\title{
Induced trees in triangle-free graphs
}

\author{
JiŘÍ MATOUŠEK ROBERT ŠŚMAL* \\ Department of Applied Mathematics and \\ Institute of Theoretical Computer Science (ITI) \\ Charles University \\ Malostranské nám. 25, 11800 Praha 1 \\ Czech Republic
}

Submitted: Nov 29, 2007; Accepted: Feb 24, 2008; Published: Mar 12, 2008

Mathematics Subject Classification: 05C55, 05C05

\begin{abstract}
We prove that every connected triangle-free graph on $n$ vertices contains an induced tree on $\exp (c \sqrt{\log n})$ vertices, where $c$ is a positive constant. The best known upper bound is $(2+o(1)) \sqrt{n}$. This partially answers questions of Erdös, Saks, and Sós and of Pultr.
\end{abstract}

\section{Introduction}

For a graph $G$, let $t(G)$ denote the maximum number of vertices of an induced subgraph of $G$ that is a tree (i.e., connected and acyclic). There are arbitrary large graphs $G$ with $t(G) \leq 2$, namely graphs in which every connected component is a clique. To rule out these trivial examples, we need to put some restrictions on $G$.

Motivated by study of forbidden configurations in Priestley spaces [1, Pultr (private communication, 2002) asked how big $t(G)$ can be if $G$ is connected and bipartite. Formally, he was interested about asymptotic properties of the function

$$
f_{B}(n)=\min \{t(G):|V(G)|=n, G \text { connected and bipartite }\}
$$

Pultr's question was the starting point of our work. However, the function $t(G)$ was studied earlier and in a more general context by Erdős, Saks, and Sós [2]. They describe the influence of the number of edges of $G$ on $t(G)$ and, more to our point, they study how small $t(G)$ can be if $\omega(G)$ is given. They observe that $t(G) \leq 2 \alpha(G)$, and this allows

${ }^{*}$ Currently on leave from Institute for Theoretical Computer Science (ITI). The paper was finished while the second author was a PIMS postdoctoral fellow at Department of Mathematics, Simon Fraser University, Burnaby, B.C. V5A 1S6, Canada. 
them to use estimates for Ramsey numbers. This way, they show that for any fixed $k>3$ there are constants $c_{1}, c_{2}$ such that

$$
c_{1} \frac{\log n}{\log \log n} \leq \min \left\{t(G):|V(G)|=n, G \nsupseteq K_{k}\right\} \leq c_{2} \log n .
$$

For $k=3$ the lower bound still applies, but the upper bound obtained by using Ramsey numbers was only $O(\sqrt{n} \log n)$ (nowadays this approach yields $O(\sqrt{n \log n})$, due to the improved lower bound on $R(k, 3)$, see [4]). We concentrate on this case $k=3$, that is we put

$$
f_{T}(n)=\min \{t(G):|V(G)|=n, G \text { connected and triangle-free }\} .
$$

Instead of applying Ramsey theory, we approach the problem directly.

It is easy to show that $f_{T}(n) \leq f_{B}(n)=O(\sqrt{n})$. The best construction we are aware of yields $f_{B}(n) \leq(2+o(1)) \sqrt{n}$; see Section [2] A simple "blow-up" construction, also presented in Section 22, shows that if $f_{T}\left(n_{0}\right)<\sqrt{n_{0}}$ for some $n_{0}$, then $f_{T}(n)=O\left(n^{1 / 2-\varepsilon}\right)$ for a positive constant $\varepsilon>0$, and similarly for $f_{B}$. Hence, $f_{T}(n)$ either is of order exactly $\sqrt{n}$, or it is bounded above by some power strictly smaller than $1 / 2$. We conjecture that the second possibility holds, and that another power of $n$ is a lower bound.

Conjecture 1.1 There are constants $0<\alpha<\beta<1 / 2$, and $c_{1}$, $c_{2}$ such that for all $n$

$$
c_{1} n^{\alpha} \leq f_{T}(n) \leq f_{B}(n) \leq c_{2} n^{\beta}
$$

The following lower bound is the main result of this paper.

Theorem 1.2 There is a constant $c>0$ such that for all $n$

$$
f_{T}(n) \geq e^{c \sqrt{\log n}}
$$

We finish the introduction by mentioning further results concerning $t(G)$. It is interesting to consider the problem of finding induced trees in (sparse) random graphs. Vega [3] shows that $t\left(G_{n, c / n}\right)=\Omega(n)$ a.s.; Palka and Ruciński [6] prove that $t\left(G_{n, c \log n / n}\right)=$ $\Theta(n \log \log n / \log n)$ a.s.

Krishnan and Ochem [5] search for values of $f_{T}(n)$ (for small $n$ ) using a computer; they succeed to find $f_{T}(n)$ for $n \leq 15$. They also extend results of [2] about the decision problem: "given a connected graph $G$ and an integer $t$, does $G$ have an induced tree with $t$ vertices?". Not only this is NP-complete for general graphs (which is proved in [2]), but it remains NP-complete even if we restrict to bipartite graphs, or to triangle-free graphs of maximum degree 4.

\section{Initial observations}

Observation $2.1 f_{B}(n) \leq(2+o(1)) \sqrt{n}$. 
Proof: It is enough to take a path with each edge replaced by a complete bipartite graph. More precisely, we take pairwise disjoint sets $V_{i}$ (for $\left.i=-(k-1), \ldots, k-1\right)$ such that $\left|V_{i}\right|=k-|i|$. We let $G$ be the graph with vertices $V=\bigcup_{|i|<k} V_{i}$ and all possible edges between $V_{i}$ and $V_{i+1}$ (for $\left.i=-(k-1), \ldots, k-2\right)$.

It is clear that if an induced tree in $G$ contains a vertex from $V_{i}$ and two vertices from $V_{i+1}$ then it contains no vertex of $V_{j}$ for $j>i+1$; similarly for $i+1$ replaced by $i-1$. Therefore any maximum induced tree is one of trees $T_{a, b}(-(k-1) \leq a<b \leq k-1$ and $b-a>1)$ : it contains all vertices from two levels, $V_{a}$ and $V_{b}$ and one vertex from each $V_{i}$ where $a<i<b$. It is easy to compute that such tree contains $2 k-1$ vertices out of the $|V|=k^{2}$; this proves $f_{B}\left(k^{2}\right) \leq 2 k-1$. If $(k-1)^{2}<n \leq k^{2}$ then we take a subgraph of $G$ to show that $f_{B}(n) \leq 2 k-1<2 \sqrt{n}+1$.

Lemma 2.2 (Blow-up construction) Let $G$ be a connected triangle-free graph and let $W \subseteq V(G)$ be a subset of $m$ vertices $(m \geq 3$ ) such that any induced tree in $G$ contains at most $t$ vertices of $W$. Then we have $f_{T}(n)=O\left(n^{\ln (t-1) / \ln (m-1)}\right)$. The same result holds with "triangle-free" replaced by "bipartite" and with $f_{T}$ replaced by $f_{B}$.

Proof: We let $W=\left\{w_{0}, \ldots, w_{m-1}\right\}$, and write $r=m-1$ and $q=t-1$ to simplify expressions. As $G$ is triangle-free it follows that $t \geq 3$, and so $q \geq 2$.

Let $T=T_{r, l}$ be a rooted tree with $l+1$ levels (counting root as one level) in which each non-leaf vertex has $r$ sons. Next, for each vertex $v$ of $T$ we take a copy $G_{v}$ of $G$ (so that distinct copies are disjoint). Whenever $v$ is a non-leaf vertex of $T$ and $u$ is its $i$-th son, we introduce an edge between $w_{i}$ in $G_{v}$ and $w_{0}$ in $G_{u}$; the resulting graph will be called $T(G)$ (see Fig. 1). Clearly this graph is triangle-free/bipartite if $G$ was triangle-free/bipartite. Moreover, $|V(T(G))|=|V(T)| \cdot|V(G)|$ and $|V(T)|=\frac{r^{l+1}-1}{r-1}=\Theta\left(r^{l}\right)$ (since $l \rightarrow \infty$ and $r \geq 2$ ).

Let $S$ be an induced subtree of $T(G)$ and put

$$
\bar{S}=\left\{v \in V(T) \mid G_{v} \text { contains a vertex of } S\right\} .
$$

By construction, $S \cap G_{v}$ is a tree in $G_{v}$ for each $v$. So the condition on $G$ implies that each vertex of $\bar{S}$ has at most $t$ neighbors in $\bar{S}$. Consequently, we have (since $q \geq 2$ )

$$
|\bar{S}| \leq 1+\sum_{i=1}^{l}(q+1) q^{i-1} \leq 1+(q+1) \frac{q^{l}-1}{q-1}=\Theta\left(q^{l}\right) .
$$

Now recall that $q, r$, and $|V(G)|$ are constants. For a given $n$, choose the smallest $l$ such that $n \leq\left|V\left(T_{r, l}(G)\right)\right|$; we have $n=\Theta\left(r^{l}\right)$. By the above considerations,

$$
f(n) \leq f\left(T_{r, l}(G)\right) \leq|V(G)| \cdot \Theta\left(q^{l}\right)=\Theta\left(r^{l \log _{r} q}\right)=\Theta\left(n^{\log _{r} q}\right),
$$

which finishes the proof. 


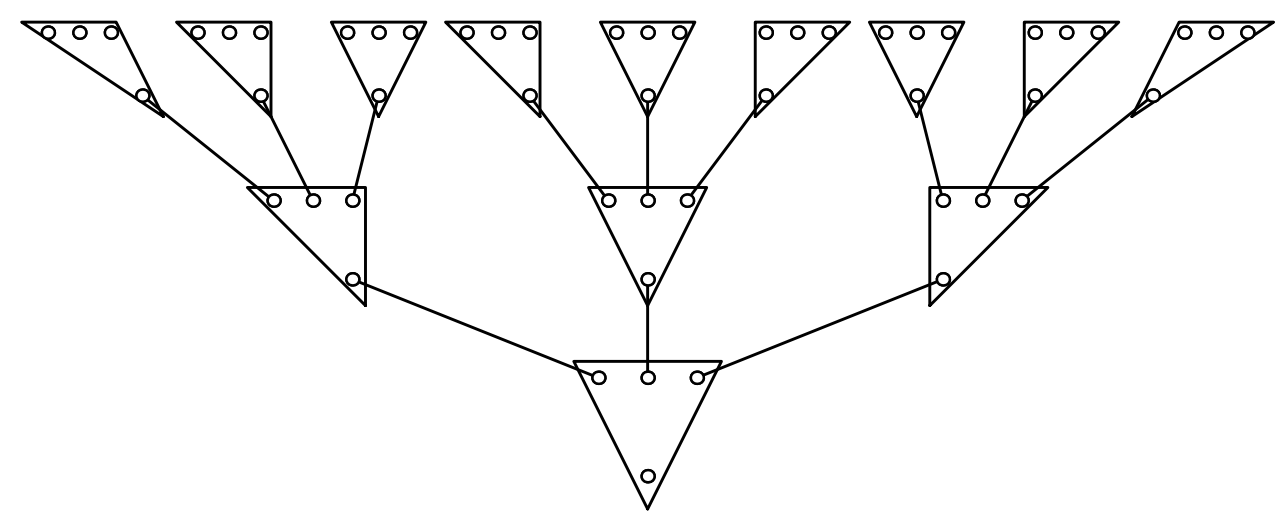

Figure 1: Graph $T_{3,2}(G)$ from the proof of Lemma 2.2.

Corollary 2.3 If $f_{T}\left(n_{0}\right)<\sqrt{n_{0}}$ for some $n_{0}$, then $f_{T}(n)=O\left(n^{1 / 2-\varepsilon}\right)$ for a positive constant $\varepsilon>0$. (The same is true for $f_{B}$.)

Proof: Let $G$ be the graph on $n_{0}$ vertices for which $t(G)=t<\sqrt{n_{0}}$. We let $W=V(G)$ and $m=n_{0}$ and apply Lemma 2.2.

As mentioned in the introduction, Krishnan and Ochem [5] search for values of $f_{T}(n)$ using a computer. This was motivated by hope that Corollary 2.3 would apply. It turns out, however, that for small $n$ Observation 2.1] gives a precise estimate even for $f_{T}(n)$ (e.g., $f_{T}(15)=7$ ); therefore Corollary 2.3 does not apply.

Remark. If we consider the construction from Lemma 2.2 for $G=K_{3}, W=V(G)$, $m=3$, and $t=2$ we recover a result of [2] that there is a graph $G$ containing triangles (but no $K_{4}$ ) such that $t(G)=O(\log n)$.

\section{Lower bound for bipartite graphs}

Here we prove a statement weaker than Theorem 1.2 - we give a bound on $f_{B}(n)$ instead of $f_{T}(n)$. The proof is simpler than that of Theorem 1.2 and it serves as an introduction to it.

We begin with a lemma about selecting induced forests of a particular kind in a bipartite graph. We introduce some terminology. Let $H$ be a bipartite graph with color classes $A$ and $B$. We will think of $A$ as the "top" class and $B$ as the "bottom" class (in a drawing of $G$ in the plane, say). We write $a=|A|$ and $b=|B|$. For a subgraph $F$ of $H$ we write $A(F)=V(F) \cap A$, we set $a(F)=|A(F)|$, and we define $B(F)$ and $b(F)$ similarly.

Whenever we say forest we actually mean an induced subgraph of $H$ that is a forest. An up-forest $F$ is a forest such that every vertex in $A(F)$ has degree (in $F$ ) precisely 1 and every vertex in $B(F)$ has degree (in $F$ ) at least 1.

A matching is a forest $F$ in which all vertices have degrees (in $F$ ) exactly 1. 


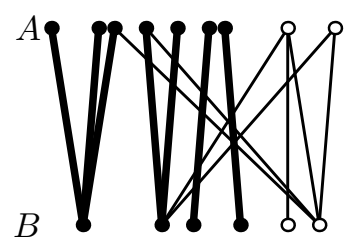

Figure 2: An up-forest

Lemma 3.1 Let $H$ be a bipartite graph with color classes $A$ and $B$ as above, let $\Delta$ be the maximum degree of $H$, and let $\eta \in(0,1)$ be a real parameter. Let us suppose that every vertex in $A$ is connected to at least one vertex in $B$. Then at least one of the following cases occurs:

(M) There is a matching with at least $(1-\eta)$ a edges.

(B) There is an up-forest $F$ with

$$
b(F) \geq \frac{\eta}{\Delta^{3}} \cdot a
$$

that is 2-branching, meaning that every vertex in $B(F)$ has degree at least 2 in $F$.
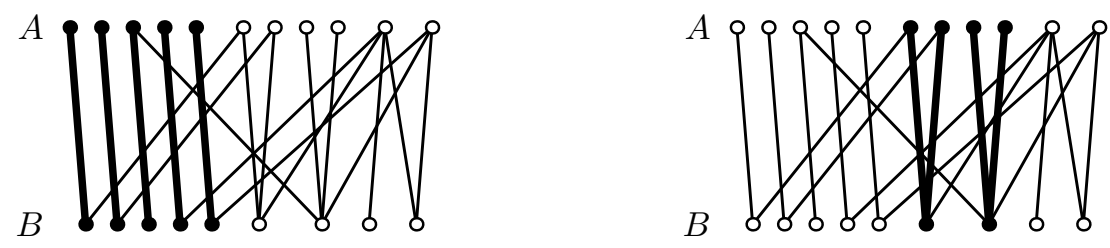

Figure 3: An illustration of Lemma 3.1

Proof. Let $B^{\prime} \subseteq B$ be the set of vertices of degree 1 in $B$. If $\left|B^{\prime}\right| \geq(1-\eta) a$ then, clearly, case $(\mathrm{M})$ occurs, so we may assume $\left|B^{\prime}\right|<(1-\eta) a$. Let $B^{\prime \prime} \subseteq B$ consist of all vertices of degree at least 2. Since every vertex in $A$ has degree at least $1,\left|E\left(H \backslash N\left(B^{\prime}\right)\right)\right| \geq \eta a$, and so $\left|B^{\prime \prime}\right| \geq(\eta / \Delta) a$.

Let us set $B_{0}=B^{\prime \prime}$ and let $F_{0}$ be an empty graph. Supposing that a set $B_{i-1} \subseteq B^{\prime \prime}$ and an up-forest $F_{i-1}$ have already been constructed with $B_{i-1} \neq \emptyset$, we construct $B_{i}$ and $F_{i}$. We let $v_{i}$ be an arbitrary vertex in $B_{i-1}$, and we let $S_{i}$ be the star formed by $v_{i}$ and all of its neighbors in $A$. We set $F_{i}=F_{i-1} \cup S_{i}$, we let $N_{i} \subseteq B$ be the neighborhood of $A\left(S_{i}\right)$, and we let $B_{i}$ be $B_{i-1} \backslash N_{i}$. The construction finishes when $B_{i}=\emptyset$, with $F_{i}$ as the resulting up-forest.

It is easy to check that this construction indeed yields an up-forest $F$ with each degree in $B(F)$ at least 2. We have $a\left(S_{i}\right) \leq \Delta$ and $\left|N_{i}\right| \leq a\left(S_{i}\right)(\Delta-1)+1$, and so in each step, at most $\left|N_{i}\right| \leq \Delta(\Delta-1)+1 \leq \Delta^{2}$ vertices are removed from $B_{i}$. Having started with at least $(\eta / \Delta) a$ vertices, we can proceed for at least $\left(\eta / \Delta^{3}\right) a$ steps, and so the resulting up-forest is as in (B). 
Now we prove the lower bound

$$
f_{B}(n) \geq e^{c \sqrt{\log n}}
$$

for a constant $c>0$.

Let $G$ be a given connected bipartite graph. We assume that $n=|V(G)|$ is sufficiently large whenever convenient. We let $t$ be the "target size" of an induced tree in $G$ we are looking for; namely, $t=\lceil\exp (c \sqrt{\log n})\rceil$. If $G$ has a vertex of degree at least $t-1$, then we can take its star for the induced tree and we are done, so we may assume that the maximum degree satisfies $\Delta \leq t-2$.

Let us fix an arbitrary vertex of $G$ as a root, and let $L_{i}$ be the set of vertices of $G$ at distance precisely $i$ from the root. All edges of $G$ go between $L_{i-1}$ and $L_{i}$ for some $i$, since an edge within some $L_{i}$ would close an odd cycle.

We may assume that $L_{t}=\emptyset$, for otherwise $G$ contains an induced path of length $t$. Hence there is a $k$ with $\left|L_{k}\right| \geq n / t$.

Let us fix such a $k$. We are going to construct sets $M_{i} \subseteq L_{i}, i=k, k-1, \ldots$, inductively, until we first reach an $i$ with $\left|M_{i}\right|=1$ (this happens for $i=0$ at the latest since $\left|L_{0}\right|=1$ ). We shall let $\ell$ be this last $i$.

Suppose that nonempty sets $M_{k}, M_{k-1}, \ldots, M_{i}$ have already been constructed, in such a way that the subgraph of $G$ induced by $M_{k} \cup \cdots \cup M_{i}$ is a forest, each of whose components intersects $M_{i}$ in at most one vertex. We are going to construct $M_{i-1}$.

Let us put $A=M_{i}, B=L_{i-1}$, and let us consider the bipartite graph $H$ induced by $A \cup B$ in $G$. Every vertex of $A$ is connected to at least one vertex in $B$. We set $\eta=\frac{1}{t}$ and apply Lemma 3.1. This yields an up-forest $F$ in $H$ as in the lemma. We define $M_{i-1}=B(F)$.

If $F$ is a matching, i.e., case (M) occurred in the lemma, we call the step from $M_{i}$ to $M_{i-1}$ a matching step. In this case, we have $\left|M_{i-1}\right| \geq\left(1-\frac{1}{t}\right)\left|M_{i}\right|$. Otherwise, $F$ is a 2-branching forest; then we call the step a branching step and we have $\left|M_{i-1}\right| \geq$ $\left|M_{i}\right| /\left(t \Delta^{3}\right) \geq\left|M_{i}\right| / t^{4}$.

Suppose that the sets $M_{k}, \ldots, M_{\ell}$ have been constructed, $\left|M_{\ell}\right|=1$. We claim that the number $b$ of branching steps in the construction is at least $c_{1} \sqrt{\log n}$ for a suitable constant $c_{1}>0$. Indeed, there are no more than $t$ matching steps, and so $1=\left|M_{\ell}\right| \geq$ $\left|M_{k}\right|(1-1 / t)^{t} t^{-4 b} \geq(n / t) e^{-1} / 2 \cdot t^{-4 b}=\Omega\left(n t^{-4 b-1}\right)$. Thus $b=\Omega(\log n / \log t)=\Omega(\sqrt{\log n})$, since $t=\lceil\exp (c \sqrt{\log n})\rceil$.

It is easy to see that $M_{k} \cup M_{k-1} \cup \cdots \cup M_{\ell}$ induces a forest in $G$. We let $T$ be the component of this forest containing the single vertex of $M_{\ell}$. Since every vertex of $M_{i-1}$, $\ell<i \leq k$, has at least one neighbor in $M_{i}$, and if the step from $M_{i}$ to $M_{i-1}$ was a branching step then each vertex of $M_{i-1}$ has at least two neighbors in $M_{i}$, it follows that $T$ has at least $2^{b}=\exp (\Omega(\sqrt{\log n}))$ vertices. This finishes the proof of the lower bound $f_{B}(n) \geq \exp (c \sqrt{\log n})$.

Remark. The above proof may seem wasteful in many respects. However, the result is tight up to the value of the constant in the exponent if we insist on selecting an induced tree "growing up" (i.e., made of up-forests for some choice of root and corresponding 
sets $L_{i}$ ). Indeed, any such induced tree in the graph $G_{r}$ in Figure 4 may contain at most two of the $r$ vertices at the topmost level of the graph. Let us put $r=\exp (c \sqrt{\log n})$ and glue copies of $G_{r}$ according to the pattern of a complete $r$-ary tree (as in the proof of Lemma 2.2), so that the resulting graph has approximately $n$ vertices (that is, the depth is $l=\Theta(\sqrt{\log n})$. We obtain a graph with all up-growing induced trees having size at most $2^{l}=\exp (O(\sqrt{\log n}))$.

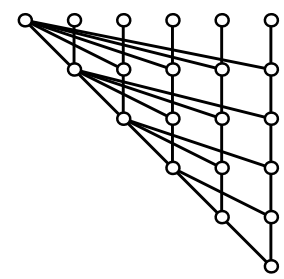

Figure 4: Graph $G_{6}$ in which all "up-growing trees" contain at most two vertices of the uppermost level.

\section{Lower bound for triangle-free graphs}

Here we prove Theorem 1.2 The scheme of the proof is very similar to the proof of the same bound for bipartite graphs in Section [3. We continue using the definitions and notation from that proof. So we decompose the given graph into the levels $L_{0}, L_{1}, \ldots, L_{r}$, $r<t$. The main difference compared to the bipartite case is that there may now be edges within the levels $L_{i}$. We will need the well-known fact that any graph on $n$ vertices with maximum degree $\Delta$ contains an independent set of size at least $n /(\Delta+1)$. We will also need the following simple modification.

Lemma 4.1 Let $\Gamma$ be a graph (not necessarily bipartite) on $n$ vertices with maximum degree $\Delta$, and let $\eta \in[0,1]$ be a real parameter. Then at least one of the following two cases occurs:

(IS) $\Gamma$ contains an independent set with at least $(1-\eta) n$ vertices.

(IM) $\Gamma$ contains an induced matching with at least $\frac{\eta}{2 \Delta} n$ edges.

Proof. We repeatedly select edges $e_{1}, e_{2}, \ldots$ of $\Gamma$; having selected $e_{i}$, we delete it and all the neighbors of its endvertices from the current graph. In each step we delete at most $2 \Delta$ vertices, so we either construct an induced matching as in (IM) or reach an edgeless graph after deleting at most $\eta n$ vertices, hence yielding an induced set as in (IS).

Proof of Theorem 1.2. We proceed similarly as in the previous section. We suppose $G$ is a given triangle-free graph on $n$ vertices (and that $n$ is big enough), we put $t=$ $\lceil\exp (c \sqrt{\log n})\rceil$. Again, we may assume $t \leq \Delta-2: G$ is triangle-free, so a star of a vertex is an induced tree. 
As before, we begin by selecting a root vertex and constructing the at most $t$ levels $L_{0}, L_{1}, \ldots$ We select $k$ such that $\left|L_{k}\right| \geq n / t$ and we will construct sets $M_{k}, M_{k-1}, \ldots$, $M_{\ell}$, such that $M_{i} \subseteq L_{i},\left|M_{\ell}\right|=1$, in such a way that their union induces a forest in $G$. In the induction step, we will either construct $M_{i-1}$ from $M_{i}$, or sometimes we will go down two levels at once, producing both $M_{i-1}$ and $M_{i-2}$.

We begin by selecting $M_{k}$ as an independent set in the subgraph induced by $L_{k}$. By the fact mentioned before Lemma 4.1 we may assume $\left|M_{k}\right| \geq\left|L_{k}\right| / t \geq n / t^{2}$.

We suppose that $M_{i}$ has already been constructed so that each component of the forest induced by $M_{k} \cup \cdots \cup M_{i}$ intersects $L_{i}$ in at most one vertex (and, in particular, $M_{i}$ is an independent set). Now we proceed as in the proof in Section 3. We let $A=M_{i}, B=L_{i-1}$, and we consider the bipartite graph $H$ induced by $A \cup B$ in $G$. We apply Lemma 3.1 to $H$ with $\eta=\frac{1}{t}$, obtaining an up-forest $F$. We set $M_{i-1}^{\prime}=B(F)$; this is not yet the final $M_{i}$ since there may be edges on $M_{i-1}^{\prime}$.

If case (B) occurred in Lemma [3.1] we have $\left|M_{i-1}^{\prime}\right| \geq\left|M_{i}\right| / t^{4}$. We let $M_{i-1}$ be an independent set of size $\left|M_{i}^{\prime}\right| /(\Delta+1) \geq\left|M_{i}\right| / t^{5}$ in the subgraph induced by $M_{i-1}^{\prime}$. We call this step a branching step.

If case (M) occurred in Lemma 3.1, we have $\left|M_{i-1}^{\prime}\right| \geq\left(1-\frac{1}{t}\right)\left|M_{i}\right|$. Then we apply Lemma 4.1 with $\eta=\frac{1}{t}$ to the graph $\Gamma$ induced in $G$ by $M_{i-1}^{\prime}$. If case (IS) applies in that lemma, we let $M_{i-1}$ be the independent set of size at least $\left(1-\frac{1}{t}\right)\left|M_{i-1}^{\prime}\right| \geq\left(1-\frac{1}{t}\right)^{2}\left|M_{i}\right|$; we call this step a matching step. Both the matching step and the branching step go one level down, from $i$ to $i-1$.

If case (IM) applies in Lemma 4.1, we define $M_{i-1}$ as the vertex set of the induced matching from the lemma. In this case we have $\left|M_{i-1}\right| \geq\left(1-\frac{1}{t}\right)\left|M_{i}\right| / t^{2}$. Note that this $M_{i-1}$ does not satisfy the inductive assumption (it is not an independent set). We are also going to construct $M_{i-2}$ in the same step, thus going from $i$ to $i-2$. To obtain $M_{i-2}$, we define another auxiliary bipartite graph, which we again call $H$ to save letters. The bottom color class $B$ is $L_{i-2}$, and the top color class $A$ is obtained by contracting the edges induced by $M_{i-1}$. More formally, we set $A=\left\{u u^{\prime} \in E(G): u, u^{\prime} \in M_{i-2}\right\}, B=L_{i-2}$, and $E(H)=\left\{\left\{u u^{\prime}, v\right\}: u, u^{\prime} \in A, v \in B, u v \in E(G)\right.$ or $\left.u^{\prime} v \in E(G)\right\}$. (Note that in this definition it can not happen that both $u v$ and $u^{\prime} v$ are edges of $G$, as $G$ is triangle-free.) We apply Lemma 3.1 with $\eta=\frac{1}{2}$, say, to $H$. In both of cases (M) and (B) we obtain an up-forest $F$ in $H$ with $b(F) \geq\left|M_{i-1}\right| /\left(32 t^{3}\right)$ (we note that $|A|=\frac{1}{2}\left|M_{i-1}\right|$ and that $H$ has maximum degree no larger than $2 t)$. We set $M_{i-2}^{\prime}=B(F)$, and finally, we select $M_{i-2}$ as an independent set of size at least $\left|M_{i-2}^{\prime}\right| / t$ in the subgraph induced by $M_{i-2}^{\prime}$. Since $G$ is triangle-free, one can check that $M_{k} \cup \cdots \cup M_{i-1} \cup M_{i-2}$ induces a forest. We have $\left|M_{i-2}\right| \geq\left|M_{i-1}\right| / 32 t^{4} \geq\left|M_{i}\right| \cdot\left(1-\frac{1}{t}\right) / 32 t^{6} \geq\left|M_{i}\right| / t^{7}$. We call this step from $M_{i}$ to $M_{i-2}$ a double-step.

By calculation similar to that in Section 3, we find that the number $b$ of branching steps and double-steps together is at least $\Omega(\sqrt{\log n})$. We again claim that the component of the forest induced by $M_{k} \cup \cdots \cup M_{\ell}$ containing the single vertex of $M_{\ell}$ has at least $2^{b}$ vertices. Indeed, if $M_{i}$ was obtained from $M_{i+1}$ by a branching step, then each vertex of $M_{i}$ has at least two successors in $M_{i+1}$. If $M_{i}$ was obtained from $M_{i+2}$ by a double-step, then each vertex $v$ of $M_{i}$ has at least one succesor in $M_{i+1}$, this is connected by an edge to 
precisely one other vertex of $M_{i+1}$, and both of these vertices have one neighbor in $M_{i+2}$; consequently $v$ has at least two successors in $M_{i+2}$. Theorem 1.2 is proved.

\section{Acknowledgment}

We would like to thank participants of a research seminar where initial steps of this work have been made, for a stimulating environment. In particular, we are indebted to Robert Babilon, Martin Bálek, Helena Nyklová, Ondra Pangrác, and Pavel Valtr for useful discussions and observations. We also want to thank the anonymous referee for helpful comments.

\section{References}

[1] Richard N. Ball and Aleš Pultr, Forbidden forests in Priestley spaces, Cah. Topol. Géom. Différ. Catég. 45 (2004), no. 1, 2-22.

[2] Paul Erdős, Michael Saks, and Vera T. Sós, Maximum induced trees in graphs, J. Combin. Theory Ser. B 41 (1986), no. 1, 61-79.

[3] Wenceslas Fernandez de la Vega, Induced trees in sparse random graphs, Graphs Combin. 2 (1986), no. 3, 227-231.

[4] Jeong Han Kim, The Ramsey number $R(3, t)$ has order of magnitude $t^{2} / \log t$, Random Structures Algorithms 7 (1995), no. 3, 173-207.

[5] Sivarama Krishnan and Pascal Ochem, Searching for induced trees, DocCourse Prague 2005, Project Report, Charles University, Prague 2005.

[6] Zbigniew Palka and Andrzej Ruciński, On the order of the largest induced tree in a random graph, Discrete Appl. Math. 15 (1986), no. 1, 75-83. 